\title{
Molecular Mapping of Fusarium Head Blight Resistance in the Spring Wheat Line ND2710
}

\author{
Mingxia Zhao, Guomei Wang, Yueqiang Leng, Humphrey Wanjugi, Pinggen Xi, \\ Michael D. Grosz, Mohamed Mergoum, and Shaobin Zhong ${ }^{\dagger}$
}

First, third, fifth, and eighth authors: Department of Plant Pathology, North Dakota State University, Fargo 58108; second, fourth, and sixth authors: Monsanto Company, St. Louis 63104; and seventh author: Department of Crop and Soil Sciences, University of Georgia, Griffin 30223. Current address of P. Xi: Guangdong Province Key Laboratory of Microbial Signals and Disease Control, South China Agricultural University, Guangzhou 510642, China.

Accepted for publication 17 March 2018.

\begin{abstract}
ND2710 is a hard red spring wheat line with a very high level of resistance to Fusarium head blight (FHB). It was selected from the progeny of a cross between ND2603 (an advanced breeding line derived from the Sumai 3/Wheaton cross) and Grandin (a spring wheat cultivar). The FHB resistance of ND2710 is presumably derived from Sumai 3 because the other parents (Grandin and Wheaton) are very susceptible to FHB. To identify and map the quantitative trait loci (QTL) for FHB resistance in ND2710, we developed a mapping population consisting of 233 recombinant inbred lines (RILs) from the cross between ND2710 and the spring wheat cultivar Bobwhite. These RILs along with their parents and checks were evaluated for reactions to FHB in three greenhouse experiments and one field experiment during 2013 to 2014. The population was also genotyped with the wheat $90 \mathrm{~K}$ iSelect single-nucleotide polymorphism (SNP) assay, and a genetic linkage map was developed with 1,373 noncosegregating SNP markers, which were distributed on all 21 wheat

chromosomes spanning 914.98 centimorgans of genetic distance. Genetic analyses using both phenotypic and genotypic data identified one major QTL ( $Q f h b . n d w p-3 B$ ) on the short arm of chromosome 3B, and three minor QTL (Qfhb.ndwp-6B, Qfhb.ndwp-2A, and Qfhb.ndwp-6A) on 6B, 2A, and $6 \mathrm{~A}$, respectively. The major QTL on $3 \mathrm{~B}$ was detected in all experiments and explained 5 to $20 \%$ of the phenotypic variation, while the three minor QTL on 6B, 2A, and 6A explained 5 to $12 \%$ phenotypic variation in at least two experiments, except for Qfhb.ndwp-2A, which was only detected in the field experiment. $Q f h b . n d w p-3 B$ and $Q f h b . n d w p-6 B$ were mapped to the genomic regions containing $F h b 1$ and $F h b 2$, respectively, confirming that they originated from Sumai 3. The additive effect of the major and minor QTL may contribute to the high level of FHB resistance in ND2710. The SNP markers closely linked to the FHB resistance QTL will be useful for marker-assisted selection of FHB resistance in wheat breeding programs.
\end{abstract}

Fusarium head blight (FHB), also known as scab, is a devastating fungal disease affecting all classes of wheat in North America and many other regions of the world (Goswami and Kistler 2004). In North America, Fusarium graminearum Schwabe (= Gibberella zeae (Schwein.) Petch) is the primary causal agent of the disease (McMullen et al. 1997). Severe FHB epidemics can cause huge economic losses in wheat production by reducing yield and downgrading grain quality due to mycotoxin contamination (Bai and Shaner 2004; McMullen et al. 1997). Mycotoxin-contaminated food and feed pose high risk to both humans and animals (Korosteleva et al. 2007; Parry et al. 1995; Pestka 2010).

Conventional agronomical methods such as adjusting planting date, crop rotations, tillage, or the use of fungicides are only partly effective to minimize damages caused by FHB. Utilization of FHB-resistant wheat varieties together with fungicide application is generally considered to be the most practical and effective strategy to control this disease (McMullen et al. 2012). Therefore, developing wheat cultivars with a high level of FHB resistance has been of high priority in wheat breeding programs worldwide over the last decade (Buerstmayr et al. 2009). Although no wheat

†Corresponding author: S. Zhong; E-mail: shaobin.zhong@ndsu.edu

Funding: This material is based upon work supported by the United States Department of Agriculture-Agricultural Research Service, under Agreement Number 59-02003-004.

*The $\boldsymbol{e}$-Xtra logo stands for "electronic extra" and indicates that one supplementary table is published online.

(c) 2018 The American Phytopathological Society varieties are immune to FHB, genotypes with relatively high levels of FHB resistance have been identified in different germplasm pools from Asia, Europe, and Latin America (Buerstmayr et al. 2009). Examples include Chinese cultivar Sumai 3 and its derivatives, Swiss cultivar Arina, and Brazilian cultivar Frontana (Buerstmayr et al. 2009; Paillard et al. 2004; Ruckenbauer et al. 2001; Schroeder and Christensen 1963). Sumai 3 and its derivatives consistently showed a high level of FHB resistance across different genetic backgrounds and environments and have been widely used in wheat breeding programs worldwide (Anderson et al. 2001; Buerstmayr et al. 2002, 2003; Yang et al. 2005; Zhou et al. 2002, 2004). Indeed, most of the recently released FHB-resistant spring wheat varieties in the North Central Region contain the FHB resistance derived from Sumai 3 (Anderson et al. 2011).

FHB resistance in wheat is a complex and quantitative trait controlled by multiple genes with major and/or minor effects (Buerstmayr et al. 2009; Liu et al. 2009). The development of various molecular markers has greatly facilitated the genetic study and detection of quantitative trait loci (QTL) for FHB resistance. To date, more than $200 \mathrm{FHB}$ resistance QTL have been identified on all 21 chromosomes of hexaploid wheat from a broad range of resistant sources; however, only 22 of them have been identified in multiple mapping populations and are considered stable QTL (Buerstmayr et al. 2009). The major QTL on 3BS (syn. Qfhs. $n d s u-3 B S$, designated as Fhbl) from Sumai 3 is the strongest and most reliable and has been proved to be consistently expressed in different genetic backgrounds (Anderson et al. 2007; McCartney et al. 2007; Waldron et al. 1999). DNA markers tightly linked to Fhbl (syn. Qfhs.ndsu-3BS) such as Xumn10 flanked by markers Xgwm533 and Xgwm493 have been routinely used for 
marker-assisted selection (MAS) of Fhbl (Liu et al. 2008). Fhbl was further fine mapped to a 1.1-Mb genomic region, with 28 candidate genes identified (Schweiger et al. 2016). The gene encoding chimeric lectin with agglutinin domains and a poreforming toxin-like domain has been reported to be Fhbl, conferring resistance to FHB (Rawat et al. 2016). The Fhb2 region on chromosome 6B was also dissected (Cuthbert et al. 2007) and six putative resistance genes were identified (Dhokane et al. 2016). However, effective markers have not been developed for other known and unknown FHB resistance QTL in wheat.

ND2710 was the first North Dakota hard red spring wheat experimental line generated in the process of introducing FHB resistance from Sumai 3 into adapted wheat varieties in the northern spring wheat region (del Blanco et al. 2003; Frohberg et al. 2004). It combines a high level of FHB resistance with relatively acceptable agronomic traits (Frohberg et al. 2004). ND2710 has been used as an FHB-resistant parent in the North Dakota State University (NDSU) spring wheat breeding program since 1994 (del Blanco et al. 2003). The objectives of this study were to verify the QTL for FHB resistance in ND2710, quantify the QTL effects using recombinant inbred lines (RILs) derived from the ND2710 $\times$ Bobwhite cross, and develop user-friendly markers for MAS of the FHB resistance in wheat breeding programs.

\section{MATERIALS AND METHODS}

Plant materials. To identify and map QTL for resistance to FHB in ND2710 (PI 633976), a mapping population (designated as BN) containing 233 RILs was developed from the cross between ND2710 and the FHB-susceptible spring wheat cultivar Bobwhite (PI 520368) using the single-seed descent method. ND2710 is a hard red spring wheat (Triticum aestivum L.) line combining a high level of FHB resistance derived from Sumai 3 with relatively acceptable agronomic traits (Frohberg et al. 2004). Grandin (PI 531005) and Alsen (PI 615543) were used as susceptible and moderately resistant checks, respectively.

Evaluation of reaction to FHB. The BN population and its parents (Bobwhite and ND2710) along with the checks were evaluated for type II resistance (resistance to fungal spread in the spikes) in three greenhouse experiments and one field environment using the procedures described by Chu et al. (2011). Greenhouse evaluations were conducted in the spring and winter seasons of 2013 (13GH1 and 13GH2) and the spring season of 2014 (14GH). Field evaluation was carried out in an FHB nursery located at Fargo, ND in 2014 (14FAR). In all experiments, the RILs were evaluated using a randomized complete block design with three replicates.

In greenhouse environments, the RILs and their parents were grown in clay pots with three plants per pot. The greenhouse was supplemented with artificial light for a 14-h photoperiod with the temperature maintained between 22 and $25^{\circ} \mathrm{C}$. The inoculum was prepared at a concentration of 100,000 spores $\mathrm{ml}^{-1}$ by mixing spores equally from four pathogenic $F$. graminearum strains collected from North Dakota (Puri and Zhong 2010). Inoculation was performed using the single-spikelet inoculation method described by Stack et al. (2002). Briefly, $10 \mu \mathrm{l}$ of spore suspension was injected into a central spikelet of a spike using a syringe at anthesis. In each pot, 8 to 10 spikes at a similar developmental stage were inoculated. Inoculated plants were placed in a room with a misting system ( $1 \mathrm{~min}$ of misting in every half hour) to facilitate disease development. After $48 \mathrm{~h}$ of incubation, the plants were moved back to the greenhouse benches. In the field experiment, each line or genotype was planted in a hill plot with 8 to 10 seeds. At anthesis, at least eight spikes at a similar developmental stage in a hill were inoculated using the single-spikelet inoculation method, as described above. The nursery was misted for $5 \mathrm{~min}$ in 15-min intervals for $12 \mathrm{~h}$ daily (4:00 P.M. to 4:00 A.M.), until 14 days after anthesis of the latest maturing lines.
For both greenhouse and field experiments, disease ratings were conducted at 21 days postinoculation. The percentage of infected spikelets on each head was recorded as FHB severity using a 1-to9 scale described by Stack and McMullen (1995) to reflect 0, 7, $14,21,33,50,67,80$, and $100 \%$ of symptomatic spikelets in an inoculated spike, respectively. The disease severity for each replicate (pot or hill) was calculated by averaging the severities of all heads.

The content of deoxynivalenol (DON) produced by $F$. graminearum in grains was determined in two greenhouse experiments (13GH2 and $14 \mathrm{GH}$ ), designated as $13 \mathrm{GH} 2-\mathrm{DON}$ and $14 \mathrm{GH}-\mathrm{DON}$, respectively. To prepare the seed samples used for the DON test, all of the inoculated spikes of each line were harvested at maturity and threshed carefully to keep scabby seed. The threshed kernels of all three replicates from each line were combined, ground into powder, and sent to the Veterinary Diagnostic Laboratory of NDSU (Fargo) for DON analysis.

Genotyping and marker development. Genomic DNA was extracted from fresh leaf tissues of the parents and RIL population $\left(\mathrm{F}_{6: 7}\right)$ using the method of Tai and Tanksley (1990) except that the leaf tissue was macerated with TissueLyser (Qiagen). DNA was diluted to approximately $50 \mathrm{ng} / \mu \mathrm{l}$ and used for genotyping with the wheat 90K iSelect assay (Wang et al. 2014). Genotypic clusters for every single-nucleotide polymorphism (SNP) were determined using the manual option of GenomeStudio (version 2011.1) with the polyploid clustering module v1.0 (Illumina), on the basis of the data from all of the described genotypes. DNA markers (UMN10, Xgwm493, $X g w m 533$, and Xgwm644) linked to Fhb1 or Fhb2 (Liu et al. 2008; Röder et al. 1998) were also used to genotype the $\mathrm{BN}$ population. Additional markers were developed for $F h b 2$ using the six candidate genes (GENE1, GENE2, GENE3, GENE4, GENE5, and GENE6) for 4-coumarate CoA ligase, basic helix-loop-helix transcription factor, glutathione S-transferase (GST), ABC transporter-4, callose synthase, and cinnamyl alcohol dehydrogenase, respectively (Dhokane et al. 2016). The gene sequences were retrieved from wheat survey sequence annotation browser on the website of URGI (https://urgi. versailles.inra.fr/gb2/gbrowse/wheat_survey_sequence_annotation/) and used for designing primer pairs using Primer3 (http://bioinfo. ut.ee/primer3-0.4.0/primer3/). These primer pairs were used in polymerase chain reaction (PCR) to amplify DNA sequences from the two parental genotypes (ND2710 and Bobwhite). PCR products amplified from two parental genotypes were sequenced by Genescript (Piscataway, NJ). Sequences from the two parental wheat genotypes were aligned against each other to identify single nucleotide polymorphism (SNP), which were transformed into cleaved amplified polymorphic sequence (CAPS) markers. These markers, together with the SNP showing polymorphism between two parents identified from $90 \mathrm{~K}$ iSelect assay, were used to genotype the mapping population.

Statistical analysis, linkage map construction, and QTL analysis. For testing distribution of disease severity and DON content in the BN population, the Shapiro-Wilk normality test was performed using PROC UNIVARIATE (SAS Institute 2011). Levene's test (Levene 1960) under the general linear model (GLM) procedure was used to test homogeneity of disease severity and DON content variances among all experiments using the SAS program, version 9.3 (SAS Institute 2011). Correlation coefficients between disease severity and DON content were calculated using the PROC CORR procedure (SAS Institute 2011). Broad-sense heritability for each trait was calculated using the restricted maximum-likelihood method in SAS (Holland et al. 2003). Heritability coefficients for single-season data were estimated using the equation $\mathrm{H}=\delta_{\mathrm{G}^{2}} /\left(\delta^{2}{ }_{\mathrm{G}}+\right.$ $\left.\delta^{2} / \mathrm{r}\right)$ and, for multiple seasons, the equation $\mathrm{H}=\delta^{2}{ }_{\mathrm{G}} /\left(\delta^{2}{ }_{\mathrm{G}}+\delta^{2}{ }_{\mathrm{GXY}} / \mathrm{y}+\right.$ $\delta^{2}{ }_{\mathrm{E}}$ /year) was used, where $\delta^{2}{ }_{\mathrm{G}}=$ genotypic variation, $\delta^{2}{ }_{\mathrm{GXY}}=$ genotype-year interaction variance, $\delta^{2} \mathrm{E}=$ residual variance, $\mathrm{y}=$ number of years, and $\mathrm{r}=$ number of replications.

A genetic linkage map was constructed using the computer program MapDisto (v1.7.7) (Lorieux 2012). The Kosambi mapping function was used and the threshold value of logarithm of odds 
(LOD) score was set at 3.0 to claim linkage between markers, with a maximum fraction of recombination at 0.30 .

The entire marker dataset was used to identify genomic regions associated with FHB resistance in the RIL population. According to the results of Levene's test, six phenotypic datasets were separately analyzed for QTL detection. Composite interval mapping was used to identify significant QTL using software QGene, v.4.3.10 (Joehanes and Nelson 2008). A QTL was claimed to be significant when a LOD value is equal or larger than 3.0. The percentage of phenotypic variance explained by a QTL and its additive effect were calculated. For the purpose of presentation, linkage groups possessing significant QTL were reevaluated using a set of nonredundant markers to generate the QTL figures. The results were confirmed to be identical to the initial results with the entire marker dataset.

To determine whether resistance QTL, environment, and interactions between them affect FHB disease severity, genotypic data of markers at each QTL peak along with disease severity data of the BN population collected from each environment were used for variance analysis through the GLM procedure in SAS, with the environment set as the random factor. The RILs in the $\mathrm{BN}$ population were divided into five groups according to the genotypes of marker loci associated with QTL detected, and Fisher's least significant difference (LSD) test was used to determine significant differences in mean FHB severities among different groups.
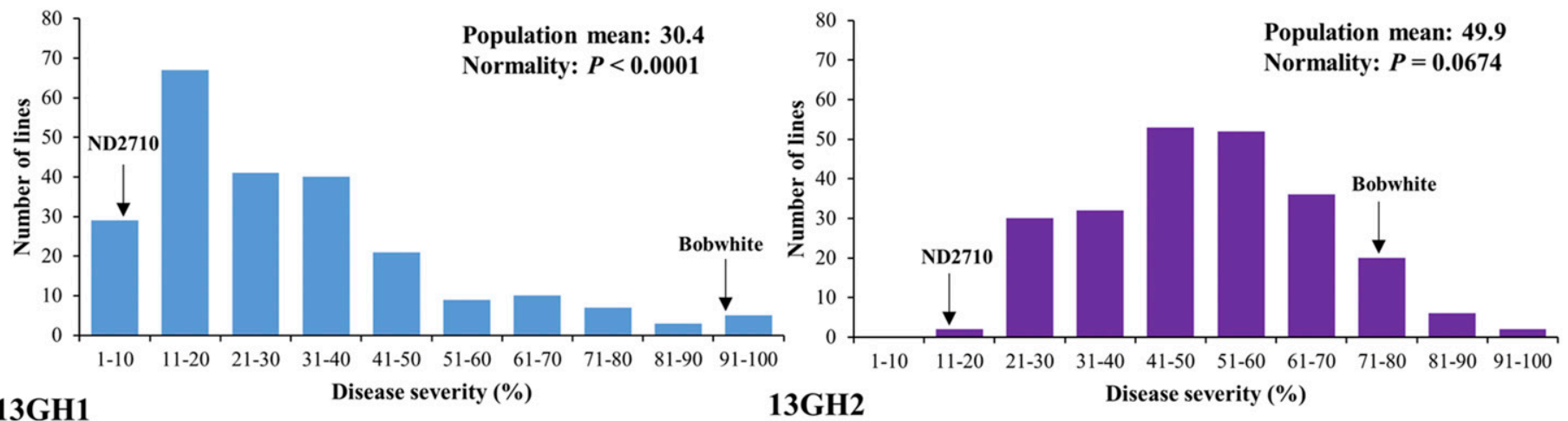

\section{GH1}

Disease severity $(\%)$

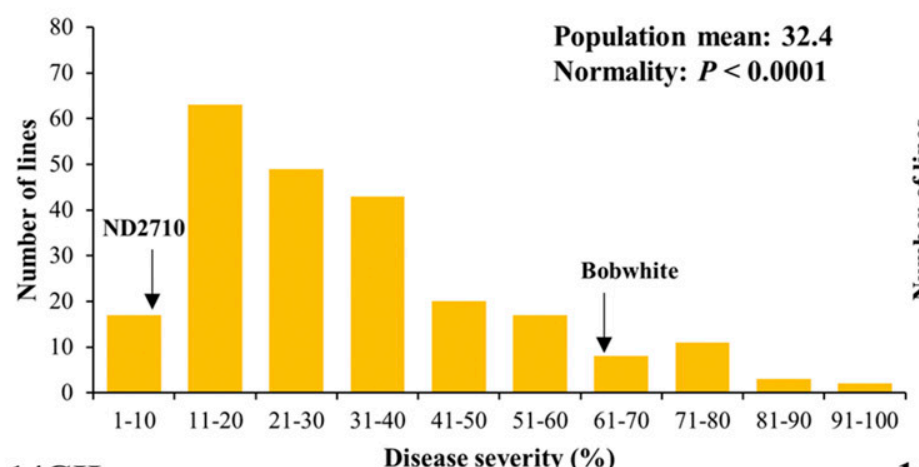

$14 \mathrm{GH}$

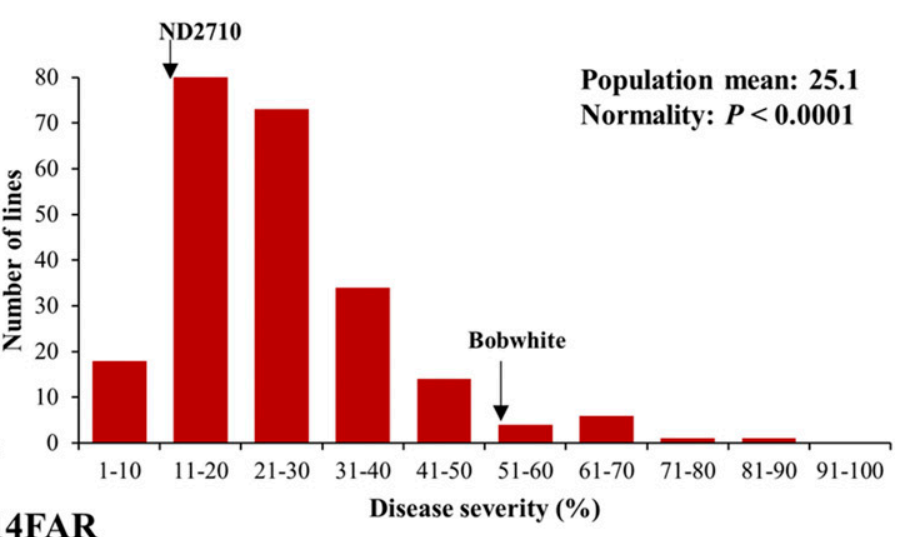

14FAR

Fig. 1. Frequency distribution of 233 recombinant inbred lines derived from the Bobwhite $\times$ ND2710 cross for Fusarium head blight (FHB) disease severity in the four environments. $13 \mathrm{GH} 1,13 \mathrm{GH} 2$, and $14 \mathrm{GH}$ represent the greenhouse experiments performed in spring and winter 2013 and spring 2014 , respectively, while 14FAR indicates field experiment performed in the FHB nursery at Fargo, ND in 2014. Arrows indicate the disease severities of parents. Normality test was performed using the PROC UNIVARIATE procedure and Shapiro-Wilk test (SAS Institute 2011).
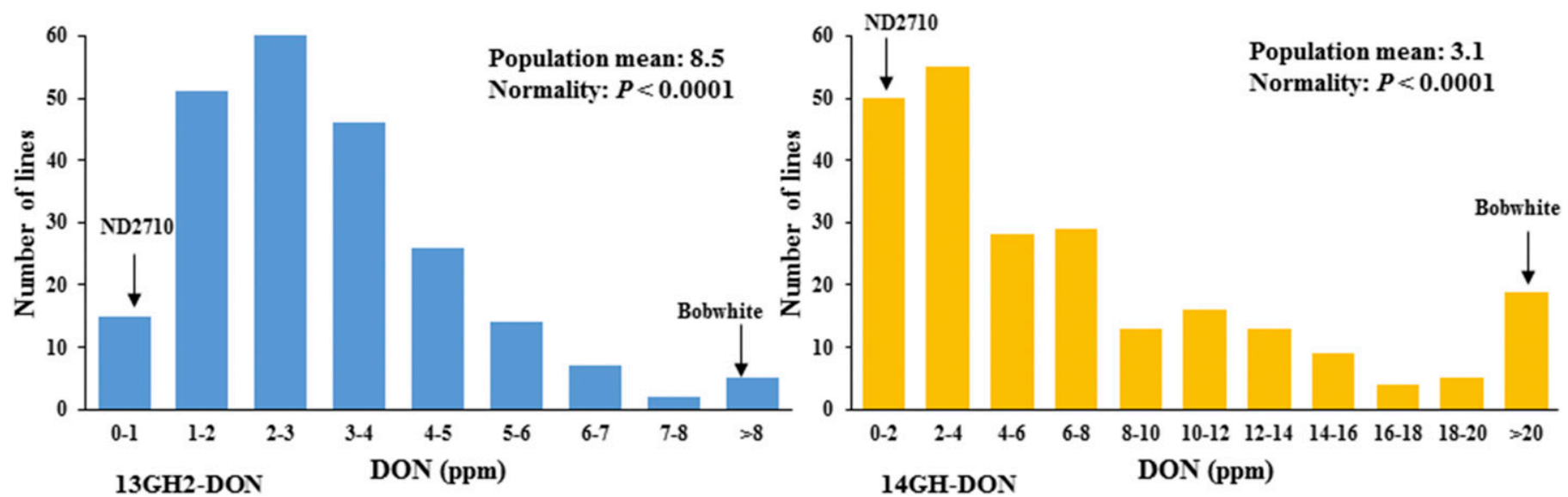

Fig. 2. Frequency distribution of 233 recombinant inbred lines derived from the cross of Bobwhite $\times$ ND2710 for deoxynivalenol (DON) content in two greenhouse experiments. 13GH2-DON and 14GH-DON represent DON tests from the experiments $13 \mathrm{GH} 2$ and $14 \mathrm{GH}$, respectively. The DON content of parents were indicated by arrows. Normality test was performed using the PROC UNIVARIATE procedure and Shapiro-Wilk test (SAS Institute 2011). 


\section{RESULTS}

FHB phenotyping across different environments. The 233 RILs in the BN population and their parents Bobwhite and ND2710 had variable reactions to FHB infection in different experiments (Fig. 1). The disease severities in $13 \mathrm{GH} 2$ were much higher than those in other experiments. In the greenhouse experiments, Bobwhite had disease severities of $91.4 \%$ in $13 \mathrm{GH} 1,74.2 \%$ in $13 \mathrm{GH} 2$, and $62.5 \%$ in $14 \mathrm{GH}$, with an average of $76.0 \%$, whereas ND2710 had disease severities of $7.8 \%$ in $13 \mathrm{GH} 1,12.5 \%$ in $13 \mathrm{GH} 2$, and $9.7 \%$ in $14 \mathrm{GH}$, with an average of $10.0 \%$ (Fig. 1). In the field nursery (14FAR), the average disease severities for Bobwhite and ND2710 were 51.8 and $10.9 \%$, respectively (Fig. 1). The distribution of disease severity of the $\mathrm{BN}$ population varied among different environments as well. The average disease severity for the population was $30.4,49.9,32.4$, and $25.1 \%$ in the experiments $13 \mathrm{GH} 1,13 \mathrm{GH} 2,14 \mathrm{GH}$, and 14FAR, respectively (Fig. 1). Over $80 \%$ of the RILs had a disease severity of $50 \%$ or less in all experiments, except for $13 \mathrm{GH} 2$, with $50 \%$ of lines having a disease severity of $50 \%$ or less (Fig. 1). DON tests (13GH2-DON and 14GH-DON) performed in the two greenhouse experiments (13GH2 and 14GH) indicated that ND2710 had a much lower DON content than Bobwhite and the RILs showed distribution patterns similar to those for the disease severity (Fig. 2).

The normality test revealed that the distribution of disease severity and DON concentration in the RIL population was significant in all experiments except for 13GH2 (Fig. 1), suggesting that the disease severity of the population in most of the FHB inoculation experiments deviated from a normal distribution. Thus, Levene's test (Levene 1960), which was less sensitive to nonnormal distribution, was used to test homogeneity of disease severity variances across all experiments. The results showed that the variances among the three greenhouse experiments and the field nursery were heterogeneous $(P<0.01, d f=3)$, and variances among three greenhouse experiments were also heterogeneous $(P<$ $0.01, d f=2)$. Only the variance between $13 \mathrm{GH} 1$ and $14 \mathrm{GH}$ was homogeneous $(P=0.36, d f=1)$. The variance between the two DON tests for greenhouse experiments was heterogeneous. There was a

TABLE 1. Correlation coefficients among Fusarium head blight traits from field and greenhouse environments of the Bobwhite $\times$ ND2710 population ${ }^{\mathrm{a}}$

\begin{tabular}{lcccccc}
\hline Experiment & $13 \mathrm{GH} 1$ & $13 \mathrm{GH} 2$ & $14 \mathrm{GH}$ & $14 \mathrm{FAR}$ & $\begin{array}{c}13 \mathrm{GH} 2- \\
\text { DON }\end{array}$ & $\begin{array}{c}14 \mathrm{GH}- \\
\mathrm{DON}\end{array}$ \\
\hline $13 \mathrm{GH}$ & $\ldots$ & $\ldots$ & $\ldots$ & $\cdots$ & $\cdots$ & $\cdots$ \\
$13 \mathrm{GH} 2$ & $0.64 * * *$ & $\ldots$ & $\ldots$ & $\cdots$ & $\cdots$ & $\cdots$ \\
$14 \mathrm{GH}$ & $0.68^{* * *}$ & $0.67 * * *$ & $\ldots$ & $\cdots$ & $\cdots$ & $\cdots$ \\
$14 \mathrm{FAR}$ & $0.43^{* * *}$ & $0.54^{* * *}$ & $0.45^{* * *}$ & $\ldots$ & $\cdots$ & $\cdots$ \\
$13 \mathrm{GH} 2-\mathrm{DON}$ & $0.47^{* * *}$ & $0.67 * * *$ & $0.49^{* * *}$ & $0.38^{* * *}$ & $\ldots$ & $\cdots$ \\
$14 \mathrm{GH}-\mathrm{DON}$ & $0.55^{* * *}$ & $0.49^{* * *}$ & $0.76^{* * *}$ & $0.43^{* * *}$ & $0.44 * * *$ & $\cdots$ \\
\hline
\end{tabular}

a Asterisks (***) indicate significance at $P<0.001 . \mathrm{GH}=$ greenhouse, $\mathrm{FAR}=$ field, and DON = deoxynivalenol. low to moderate correlation between measured FHB traits among environments, ranging from 0.38 to 0.76 (Table 1 ). The correlation coefficient was 0.67 between disease severity of $13 \mathrm{GH} 2$ and $13 \mathrm{GH} 2-\mathrm{DON}$ and 0.76 between disease severity of $14 \mathrm{GH}$ and 14GH-DON, each being highly significant $(P<0.0001)$, suggesting that RILs with a low disease severity usually had a low DON concentration in the greenhouse.

The heritability for disease severity and DON concentration were calculated. Heritability for disease severity was high, ranging from 0.70 to 0.99 for four experiments, with an overall mean of 0.74 , whereas it was low for DON concentration, with a value of 0.30 .

Linkage map construction. In total, 7,413 polymorphic SNP markers were identified in the $\mathrm{BN}$ population using the $90 \mathrm{~K}$ iSelect assays. After removal of cosegregating markers, 1,370 unique SNP markers were used for further genetic analysis. In addition, three simple-sequence repeat (SSR) markers (Xumn10, Xgwm493, and Xgwm533) associated with Fhb1, one SSR marker (Xgwm644) linked to $F h b 2$, and two CAPS markers (Fhb2-CAPS 3 and Fhb2-CAPS6) developed from two candidate genes (GENE3 and GENE6) for $F h b 2$ were used to genotype the mapping population. With a total of 1,376 markers, a genetic linkage map was developed, which consisted of 35 linkage groups. In all, 8 of the linkage groups were assigned to genome A, 11 to genome B, and 16 to genome D (Supplementary Table S1). The total genetic map length was 914.98 centimorgans $(\mathrm{cM})$, partitioned into $385.43 \mathrm{cM}$ for genome A, $384.74 \mathrm{cM}$ for genome B, and $144.82 \mathrm{cM}$ for genome D. Average distance between markers was $0.66 \mathrm{cM}$.

QTL analysis for FHB resistance and DON content. QTL analysis of six datasets (13GH1, 13GH2, 14GH, 14FAR, 13GH2DON, and 14GH-DON) led to identification of four ND2710derived QTL for FHB resistance on chromosome 3B, 6B, 2A, and $6 \mathrm{~A}$, respectively (Table 2). Qfhb.ndwp-3B peaked at marker SNP4343 was detected from all datasets, explaining 5 to $20 \%$ of the phenotypic variation, with an LOD value between 2.8 and 11.5 (Fig. 3; Table 2). Qfhb.ndwp-6B peaked at marker Fhb2-CAPS3 was detected from $13 \mathrm{GH} 1,14 \mathrm{GH}$, and $14 \mathrm{GH}-\mathrm{DON}$, explaining 6,12 , and $7 \%$ of the phenotypic variation, respectively. $Q$ fhb.ndwp- $2 A$ peaked at $S N P 79083$ was only detected from the field experiment (14FAR), explaining 6\% of phenotypic variation. Qfhb.ndwp-6A peaked at marker SNP74620 was detected from $13 \mathrm{GH} 2$ and $13 \mathrm{GH} 2-$ DON, explaining 5 and $8 \%$ of phenotypic variation, respectively. The QTL for resistance to FHB and DON accumulation on 3BS, 6BS, and 6AL were coincident (Fig. 3; Table 2).

Effects of QTL on type II resistance and DON accumulation. Under greenhouse (13GH1, 13GH2, and 14GH) and field (14FAR) conditions, the average disease severity of the RILs carrying the ND2710 alleles at SNP4343 on 3B and Fhb2-CAPS3 on 6B were 17.4 and $19.6 \%$, respectively, while the average disease severity of the RILs carrying the Bobwhite alleles were 45.4 and $30.2 \%$, respectively. In the two DON tests, the average DON content for RILs carrying ND2710 alleles at SNP4343 on 3B and Fhb2-CAPS3 on $6 \mathrm{~B}$ were 2.3 and $3.7 \mathrm{ppm}$, respectively, while the average DON

TABLE 2. Quantitative trait loci associated with Fusarium head blight (FHB) resistance in the Bobwhite $\times$ ND2710 population ${ }^{\mathrm{a}}$

\begin{tabular}{|c|c|c|c|c|c|c|c|c|c|c|c|c|}
\hline \multirow[b]{2}{*}{ Experiment $^{\mathrm{b}}$} & \multicolumn{3}{|c|}{ Qfhb.ndwp-3B } & \multicolumn{3}{|c|}{$Q f h b . n d w p-6 B$} & \multicolumn{3}{|c|}{ Qfhb.ndwp-2A } & \multicolumn{3}{|c|}{ Qfhb.ndwp-6A } \\
\hline & $\overline{\text { LOD }}$ & $R^{2}$ & $\overline{\text { Add }}$ & LOD & $R^{2}$ & $\overline{\text { Add }}$ & $\overline{\text { LOD }}$ & $R^{2}$ & $\overline{\text { Add }}$ & LOD & $R^{2}$ & $\overline{\text { Add }}$ \\
\hline $13 \mathrm{GH} 1$ & 6.3 & 0.12 & -7.2 & 3.3 & 0.06 & -5.7 & $\ldots$ & $\ldots$ & $\ldots$ & $\ldots$ & $\ldots$ & $\ldots$ \\
\hline $13 \mathrm{GH} 2$ & 11.5 & 0.20 & -7.3 & $\ldots$ & $\ldots$ & $\ldots$ & $\ldots$ & $\ldots$ & $\ldots$ & 2.7 & 0.05 & -3.9 \\
\hline 14FAR & 4.9 & 0.09 & -4.0 & $\ldots$ & $\ldots$ & $\ldots$ & 3.3 & 0.06 & -3.5 & $\ldots$ & $\ldots$ & $\ldots$ \\
\hline 13GH2-DON & 3.9 & 0.07 & -0.5 & $\ldots$ & ... & $\ldots$ & $\ldots$ & $\ldots$ & $\ldots$ & 4.1 & 0.08 & -0.5 \\
\hline $14 \mathrm{GH}-\mathrm{DON}$ & 2.8 & 0.05 & -3.3 & 3.9 & 0.07 & -4.1 & $\ldots$ & $\ldots$ & $\ldots$ & $\ldots$ & $\ldots$ & $\ldots$ \\
\hline
\end{tabular}

a The critical logarithm of odds (LOD) threshold of 3.0 for the 0.05 level of probability was obtained through a permutation test with 1,000 iterations. Add $=$ additive effect. A negative value indicates an increasing allele for disease severity derived from Bobwhite; then, the resistance effects were derived from ND2710.

b $13 \mathrm{GH} 1,13 \mathrm{GH} 2$, and 14GH were experiments conducted in the greenhouse in spring and winter 2013 and spring 2014, respectively; 14FAR was the experiment conducted in the field FHB nursery at Fargo, ND in 2014; and 13GH2-DON and 14GH-DON represent the content of deoxynivalenol (DON) produced by Fusarium graminearum calculated from two greenhouse experiments $13 \mathrm{GH} 2$ and $14 \mathrm{GH}$. 
content of RILs carrying the Bobwhite alleles were 3.7 and 17.1 ppm, respectively. For the other two markers on $2 \mathrm{~A}$ and $6 \mathrm{~A}$, the average disease severity of the RILs with ND2710 alleles in greenhouse and field experiments were 27.9 and $20.3 \%$, compared with 34.9 and $30.1 \%$ for those carrying the corresponding Bobwhite alleles. The average DON contents of the RILs with ND2710 alleles were 2.4 and $6.8 \mathrm{ppm}$, compared with 3.9 and $13.1 \mathrm{ppm}$ for those with the Bobwhite alleles (Fig. 4). The lower average disease severity and DON content in RILs with ND2710 alleles than in those with Bobwhite alleles confirmed that all favorable alleles for FHB resistance were contributed by ND2710. Qfhb. $n d w p-3 B$ contributed the largest effect on FHB resistance, followed by $Q f h b$. $n d w p-6 B$.

To elucidate the effect of single and combined QTL on FHB response, the RILs were divided into five groups: group 1 (G1 = 16 RILs) carried the ND2710 alleles at the peak marker loci associated with all four QTL detected, group 2 (G2 = 7 RILs) contained the
ND2710 alleles associated with Qfhb.ndwp-3B and Qfhb.ndwp-6B, group 3 (G3 = 16 RILs) carried the ND2710 allele associated with Qfhb.ndwp-3B only, group 4 (G4 = 8 RILs) carried the ND2710 allele associated with $Q f h b . n d w p-6 B$ only, group 5 (G5 = 17 RILs) carried the ND2710 alleles associated with the two minor QTL Qfhb.ndwp-2A and Qfhb.ndwp-6A, and group 6 (G6 = 15 RILs) carried the Bobwhite alleles for all four QTL. For greenhouse experiments (GH_MEAN), the average disease severities of G1 and G2 were significantly lower (LSD, $\alpha=0.05$ ) than those of G4, G5, and G6, while there were no significant differences among G1, G2, and G3 (Fig. 4). G6 showed significantly higher disease severity than the other groups. For the field experiment, G1 and G2 showed significantly lower disease severity than G4 and G6, but there were no significant differences either between G1 and G2, or between G4 and G6. G3 and G5 showed a similar level of disease severity. For the DON tests, G1 and G2 had a significantly lower DON content than G6 but the differences among G1, G2, and G3
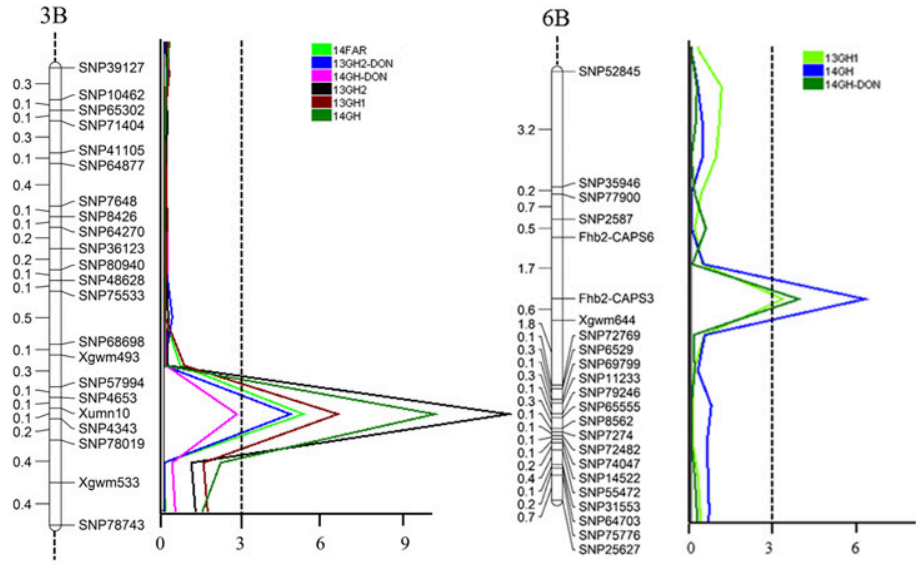

$2 \mathrm{~A}$

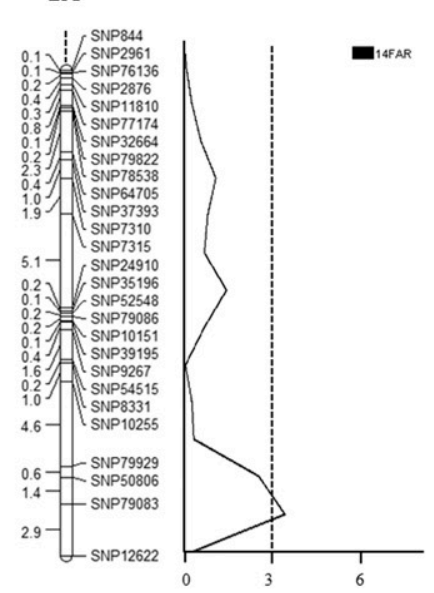

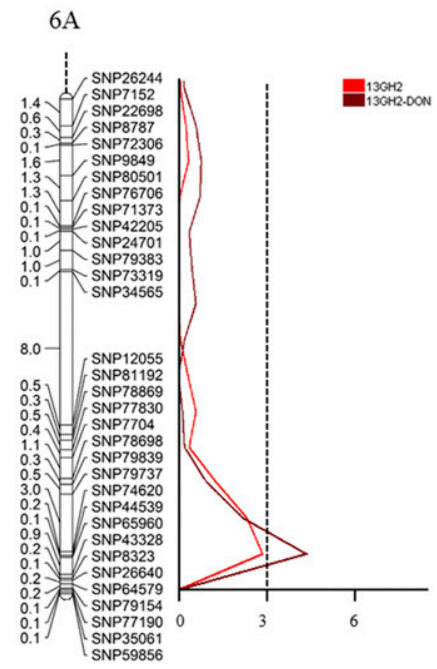

Fig. 3. Regions of linkage maps for chromosome 3B, 6B, 2A, and 6A harboring quantitative trait loci (QTL) for Fusarium head blight resistance detected in the Bobwhite $\times$ ND2710 (BN) population. All QTL were derived from the resistant parent ND2710. The centimorgan distances between marker loci are indicated on the left sides, while the positions of marker loci are marked on the right sides of the linkage maps. The logarithm of the odds significance threshold of 3.0 is represented by a vertical dotted line. Different lines indicate greenhouse seasons (13GH2, 13GH1 and 14GH), the field experiment (14FAR), and greenhouse experiments that determined content of deoxynivalenol (DON) (13GH2-DON and 14GH-DON).
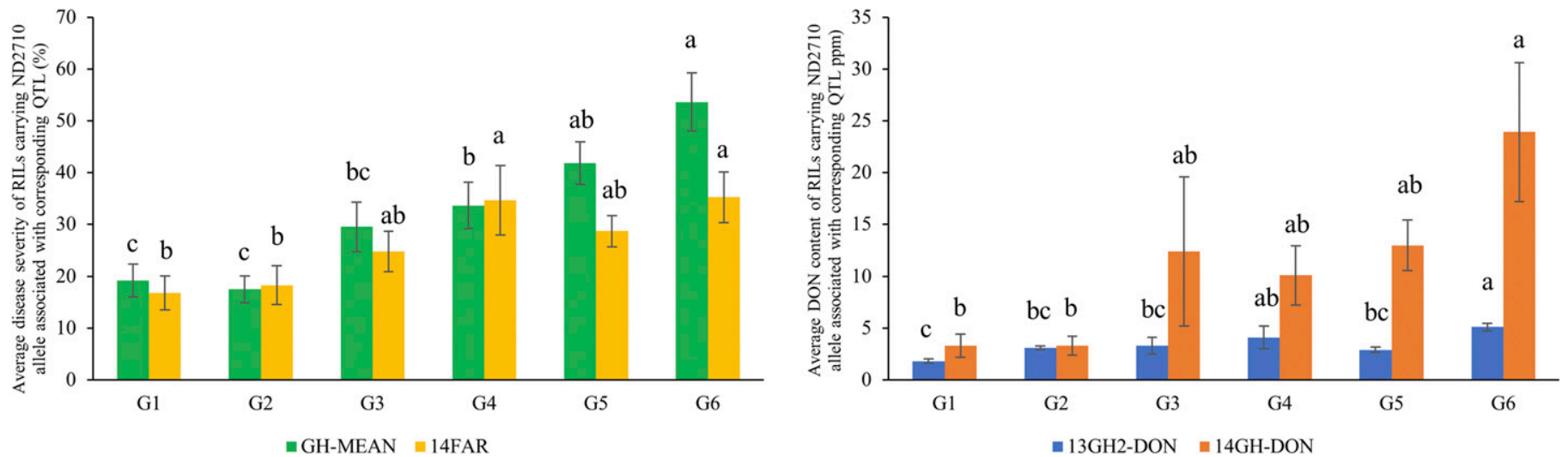

Fig. 4. Effect of quantitative trait loci (QTL) on Fusarium head blight (FHB) severity and deoxynivalenol (DON) content. To elucidate the effect of single and combined QTL on FHB response, the recombinant inbred lines (RILs) were divided into six groups: group 1 (G1) contained 16 RILs with the ND2710 alleles associated with all four QTL, group 2 (G2) contained 7 RILs carrying the ND2710 alleles associated with the 3B and 6B QTL only, group 3 (G3) contained 16 RILs carrying the ND2710 allele associated with the 3B QTL, group 4 (G4) contained 8 RILs carrying the ND2710 allele associated with 6B, group 5 (G5) contained 17 RILs harboring the two minor QTL on 2A and 6A, and group 6 (G6) contained 15 RILs carrying the Bobwhite alleles for all four QTL. GH-Mean = mean FHB severity of each group across the greenhouse experiments, 14FAR = mean FHB severity of each group in the field experiment in the Fargo location, $13 \mathrm{GH} 2-\mathrm{DON}=$ mean DON content of each group from the experiment 13GH2, and 14GH-DON = mean DON content of each group from the experiment $14 \mathrm{GH}$. Fisher's least significant difference test was used to determine significant differences among means of different groups. The same letters above the histogram indicate no significant difference at the 0.05 level of probability. 
were not significant whereas the difference between G5 and G6 was significant.

\section{DISCUSSION}

In this study, we genotyped and phenotyped the RIL population derived from the cross between ND2710 and Bobwhite, and identified four QTL for FHB resistance contributed by the hard-red spring wheat line ND2710. These QTL were mapped to chromosomes 3BS, 6BS, 2AS, and 6AL and saturated with SNP markers, which will be useful for MAS of the FHB resistance QTL from ND2710.

The QTL on 3BS and 6BS in ND2710 were mapped to the same genomic regions as $F h b 1$ and $F h b 2$, respectively. Considering the fact that ND2710 was a progeny of crosses with Sumai 3 in the pedigree (Frohberg et al. 2004) and SSR markers for Fhbl and Fhb2 mapped to the QTL regions in the BN population, the high level of FHB resistance in ND2710 was mainly due to these two QTL derived from Sumai 3, although the other two minor QTL may have some effect on the resistance as well. $F h b 1$ and $F h b 2$ were first reported in Sumai 3 (Waldron et al. 1999) and Ning 7840 (Bai et al. 1999), and also detected in many different wheat genotypes related to Sumai 3 (Buerstmayr et al. 2009; Liu et al. 2009). Due to the large effect on FHB resistance, Fhbl was fine mapped as a single Mendelian gene within a 1.2-cM interval (Cuthbert et al. 2006). Liu et al. (2008) developed an SSR marker (Xumn10), which has been widely used to predict the presence of $F h b l$ in various genetic studies and in breeding programs. Recently, genome sequencing, gene annotation, and function characterization of the $F h b l$ region have led to identification and cloning of the candidate gene responsible for the FHB resistance (Rawat et al. 2016; Schweiger et al. 2016). These studies provide valuable resources to further understand the mechanism of the FHB resistance gene and accelerate its application in wheat breeding programs.

Among the six candidate genes identified by Dhokane et al. (2016) for Fhb2, two markers (Fhb2-CAPS3 and Fhb2-CAPS6) were developed and mapped to the $Q f h b . n d w p-6 B$ region in the population derived from the cross between Bobwhite and ND2710. Interestingly, the marker Fhb2-CAPS3 developed from the gene for GST is localized at the peak of the Fhb2-QTL region. It should be a useful additional marker for MAS of the Fhb2 QTL in wheat breeding programs. GST catalyze the conjugation of electrophilic molecules to glutathione and have been implicated for detoxification of DON in barley (Gardiner et al. 2010). Several other studies also indicated that GST genes play a role in resistance to fungal plant pathogens (Dean et al. 2003, 2005; Han et al. 2016). Dhokane et al. (2016) speculated that GST is involved in FHB resistance by detoxification of DON and reducing the pathogenicity of the pathogen. However, further studies are needed to characterize the function of GST in FHB resistance and to clone and characterize the candidate genes for $F h b 2$.

The QTL (Qfhb.ndwp-2A) on 2A was only detected in the field inoculation experiment, whereas $Q f h b . n d w p-6 A$ was detected from one greenhouse experiment and the corresponding DON test. They are considered minor QTL, which are inconsistent among different environmental conditions. However, these minor QTL are still important for further improvement of FHB resistance in wheat cultivars because commercially released spring wheat cultivars with Fhbl alone or even with Fhbl and Fhb2 combined did not have the same high and stable level of FHB resistance as ND2710 (Anderson et al. 2011; Frohberg et al. 2006). QTL for FHB resistance on chromosome $2 \mathrm{~A}$ have been reported in several sources; they were associated with either type II resistance or DON content reduction and explained 3 to $26.7 \%$ of the phenotypic variation in different experiments (Anderson et al. 2001; Gervais et al. 2003; Paillard et al. 2004; Steiner et al. 2004; Waldron et al. 1999; Zhou et al. 2002). Qfhb. $n d w p-2 A$ in ND2710 peaked at $41.7 \mathrm{cM}$ on the genetic map of this study, which is approximately at $38.7 \mathrm{cM}$ on the $9 \mathrm{~K}$ consensus SNP map (Cavanagh et al. 2013); thus, this QTL may be the same as the 2A QTL identified from winter wheat cultivar Freedom, with confidence intervals (CI) between 39.5 and $43.6 \mathrm{cM}$ (Gupta et al. 2001), and the 2A QTL from Chinese spring wheat cultivar Ning7840, with CI between 29.1 and $53.6 \mathrm{cM}$ (Zhou et al. 2002). All three QTL previously identified on $2 \mathrm{~A}$ condition type II resistance.

FHB resistance QTL were previously identified on chromosome 6AS from the U.S. wheat line ND2603 (Anderson et al. 2001), French bread wheat cultivar Apache (Holzapfel et al. 2008), German winter wheat cultivar Dream (Häberle et al. 2007; Schmolke et al. 2005), and U.K. winter wheat cultivar Spark (Gosman et al. 2007). These reported 6AS QTL condition type II resistance and explained 7.8 to $19 \%$ of phenotypic variation. ND2710 has a pedigree with ND2603 but no QTL on 6AS was identified in this study; instead, a QTL on 6AL was detected from dataset $13 \mathrm{GH} 2$ and the corresponding DON test 13GH2-DON. The 6AL QTL may be novel.

Over 200 QTL for FHB resistance have been identified from different resistance sources worldwide and they confer one or more of type I, II, III, and IV resistance to FHB (Buerstmayr et al. 2009). However, most of the QTL are responsible for one type of resistance, whereas some of them confer more than one type of resistance (Buerstmayr et al. 2009; Liu et al. 2009). For example, QTL identified from Sumai3, CJ9306, Wangshuibai, Ernie, and Arina condition both type II and III resistance (Abate et al. 2008; Draeger et al. 2007; Handa et al. 2008; Jiang et al. 2007a,b; Yu et al. 2008). In the present study, Qfhb.ndwp-2A conditioned type II resistance, while the QTL on chromosome $3 \mathrm{~B}, 6 \mathrm{~A}$, and $6 \mathrm{~B}$ were associated with both type II and II resistances to FHB and DON accumulation. These results are in line with previous reports showing that QTL identified in various sources may contribute to one or more of the four currently recognized types of FHB resistance (Buerstmayr et al. 2009).

In summary, our data showed that FHB resistance in ND2710 was contributed by a combination of four QTL. Three of them were reported previously in different germplasms whereas one QTL was identified to be novel. The effects of these QTL were additive. The QTL on chromosome 3B showed a major effect on FHB resistance and DON reduction and was consistently detected in multiple experiments. The RILs combining ND2710 alleles at the QTL on $3 \mathrm{~B}$ and $6 \mathrm{~B}$ had significantly lower disease severity and DON content than RILs without these alleles in both greenhouse and field experiments. ND2710 had the same high level of FHB resistance as Sumai 3 but its agronomic traits were much closer to those of North Dakota varieties (Frohberg et al. 2004). It has been a very useful source for improving wheat FHB resistance, especially in the Northern Great Plains (del Blanco et al. 2003) (unpublished data). The identified DNA markers associated with the four QTL will further facilitate the selection and use of FHB resistance from ND2710 in breeding programs.

\section{ACKNOWLEDGMENTS}

We thank J. Mullins, R. Wang, S. Shrestha, Poudel, L. Sun, and Q. Li for assistance in greenhouse and field experiments. This is a cooperative project with the U.S. Wheat \& Barley Scab Initiative. Any opinions, findings, conclusions, or recommendations expressed in this publication are those of the authors and do not necessarily reflect the view of the U.S. Department of Agriculture.

\section{LITERATURE CITED}

Abate, Z. A., Liu, S., and McKendry, A. L. 2008. Quantitative trait loci associated with deoxynivalenol content and kernel quality in the soft red winter wheat 'Ernie'. Crop Sci. 48:1408-1418.

Anderson, J. A., Chao, S., and Liu, S. 2007. Molecular breeding using a major QTL for Fusarium head blight resistance in wheat. Crop Sci. Abstr. 47:S-112-S119.

Anderson, J. A., Glover, K., and Mergoum, M. 2011. Successful adoption of spring wheat cultivars with moderate resistance to FHB by growers in the North Central Region. in Proc. 2011 Natl. Fusarium Head Blight Forum. 
S. Canty, A. Clark, A. Anderson-Scully, D. Ellis, and D. Van Sanford, eds. U.S. Wheat and Barley Scab Initiative.

Anderson, J. A., Stack, R. W., Liu, S., Waldron, B. L., Fjeld, A. D., Coyne, C., Moreno-Sevilla, B., Fetch, J. M., Song, Q. J., Cregan, P. B., and Frohberg, R. C. 2001. DNA markers for Fusarium head blight resistance QTLs in two wheat populations. Theor. Appl. Genet. 102:1164-1168.

Bai, G., Kolb, F. L., Shaner, G., and Domier, L. L. 1999. Amplified fragment length polymorphism markers linked to a major quantitative trait locus controlling scab resistance in wheat. Phytopathology 89:343-348.

Bai, G., and Shaner, G. 2004. Management and resistance in wheat and barley to Fusarium head blight. Annu. Rev. Phytopathol. 42:135-161.

Buerstmayr, H., Ban, T., and Anderson, J. A. 2009. QTL mapping and markerassisted selection for Fusarium head blight resistance in wheat: A review. Plant Breed. 128:1-26.

Buerstmayr, H., Lemmens, M., Hartl, L., Doldi, L., Steiner, B., Stierschneider, M., and Ruckenbauer, P. 2002. Molecular mapping of QTLs for Fusarium head blight resistance in spring wheat. I. Resistance to fungal spread Type II resistance. Theor. Appl. Genet. 104:84-91.

Buerstmayr, H., Steiner, B., Hartl, L., Griesser, M., Angerer, N., Lengauer, D., Miedaner, T., Schneider, B., and Lemmens, M. 2003. Molecular mapping of QTLs for Fusarium head blight resistance in spring wheat. II. Resistance to fungal penetration and spread. Theor. Appl. Genet. 107:503-508.

Cavanagh, C. R., Chao, S., Wang, S., Huang, B. E., Stephen, S., Kiani, S., Forrest, K., Saintenac, C., Brown-Guedira, G. L., Akhunova, A., and See, D. 2013. Genome-wide comparative diversity uncovers multiple targets of selection for improvement in hexaploid wheat landraces and cultivars. Proc. Natl. Acad. Sci. USA 110:8057-8062.

Chu, C., Niu, Z., Zhong, S., Chao, S., Friesen, T. L., Halley, S., Elias, E. M., Dong, Y., Faris, J. D., and Xu, S. S. 2011. Identification and molecular mapping of two QTLs with major effects for resistance to Fusarium head blight in wheat. Theor. Appl. Genet. 123:1107-1119.

Cuthbert, P. A., Somers, D. J., and Brulé-Babel, A. 2007. Mapping of Fhb2 on chromosome 6BS: A gene controlling Fusarium head blight field resistance in bread wheat (Triticum aestivum L.). Theor. Appl. Genet. 114: 429-437.

Cuthbert, P. A., Somers, D. J., Thomas, J., Cloutier, S., and Brulé-Babel, A. 2006. Fine mapping Fhbl, a major gene controlling Fusarium head blight resistance in bread wheat (Triticum aestivum L.). Theor. Appl. Genet. 112:1465-1472.

Dean, J. D., Goodwin, P. H., and Hsiang, T. 2003. Colletotrichum gloeosporioides infection induces differential expression of glutathione S-transferase genes in Malva pusilla. Funct. Plant Biol. 30:821-828.

Dean, J. D., Goodwin, P. H., and Hsiang, T. 2005. Induction of glutathione S-transferase genes of Nicotiana benthamiana following infection by Colletotrichum destructivum and C. orbiculare and involvement of one in resistance. J. Exp. Bot. 56:1525-1533.

del Blanco, I., Frohberg, R., Stack, R., Berzonsky, W., and Kianian, S. 2003. Detection of QTL linked to Fusarium head blight resistance in Sumai 3-derived North Dakota bread wheat lines. Theor. Appl. Genet. 106:1027-1031.

Dhokane, D., Karre, S., Kushalappa, A. C., and McCartney, C. 2016. Integrated metabolo-transcriptomics reveals Fusarium head blight candidate resistance genes in wheat QTL-Fhb2. PLoS One 11:e0155851.

Draeger, R., Gosman, N., Steed, A., Chandler, E., Thomsett, M., Schondelmaier, J., Buerstmayr, H., Lemmens, M., Schmolke, M., Mesterhazy, A., and Nicholson, P. 2007. Identification of QTLs for resistance to Fusarium head blight, DON accumulation and associated traits in the winter wheat variety Arina. Theor. Appl. Genet. 115:617-625.

Frohberg, R. C., Stack, R. W., and Mergoum, M. 2004. Registration of spring wheat germplasm ND2710 resistant to Fusarium head blight. Crop Sci. 44: 1498-1500.

Frohberg, R. C., Stack, R. W., Olson, T., Miller, J. D., and Mergoum, M. 2006. Registration of 'Alsen' wheat. Crop Sci. 46:2311-2312.

Gardiner, S. A., Boddu, J., Berthiller, F., Hametner, C., Stupar, R. M., Adam, G., and Muehlbauer, G. J. 2010. Transcriptome analysis of the barleydeoxynivalenol interaction: Evidence for a role of glutathione in deoxynivalenol detoxification. Mol. Plant-Microbe Interact. 23:962-976.

Gervais, L., Dedryver, F., Morlais, J. Y., Bodusseau, V., Negre, S., Bilous, M., Groos, C., and Trottet, M. 2003. Mapping of quantitative trait loci for field resistance to Fusarium head blight in an European winter wheat. Theor. Appl. Genet. 106:961-970.

Gosman, N., Bayles, R., Jennings, P., Kirby, J., and Nicholson, P. 2007. Evaluation and characterization of resistance to Fusarium head blight caused by Fusarium culmorum in UK winter wheat cultivars. Plant Pathol. 56:264-276.

Goswami, R. S., and Kistler, H. C. 2004. Heading for disaster: Fusarium graminearum on cereal crops. Mol. Plant Pathol. 5:515-525.

Gupta, A., Lipps, P. E., Campbell, K. G., and Sneller, C. H. 2001. Identification of QTL associated with resistance to FHB in Ning 7840 and Freedom. Page 180 in: Proc. 2001 Natl. Fusarium Head Blight Forum. Michigan State University, East Lansing.
Häberle, J., Schmolke, M., Schweizer, G., Korzun, V., Ebmeyer, E., Zimmermann, G., and Hartl, L. 2007. Effects of two major Fusarium head blight resistance QTL verified in a winter wheat backcross population. Crop Sci. 47:1823-1831.

Han, Q., Chen, R., Yang, Y., Cui, X., Ge, F., Chen, C., and Liu, D. 2016. A glutathione S-transferase gene from Lilium regale Wilson confers transgenic tobacco resistance to Fusarium oxysporum. Sci. Hortic. (Amsterdam) 198:370-378.

Handa, H., Namiki, N., Xu, D., and Ban, T. 2008. Dissecting of the FHB resistance QTL on the short arm of wheat chromosome 2D using a comparative genomic approach: From QTL to candidate gene. Mol. Breed. 22: 71-84.

Holland, J. B., Nyquist, W. E., and Cervantes-Martinez, C. T. 2003. Estimating and interpreting heritability for plant breeding: An update. Plant Breed. Rev. 22:9-111.

Holzapfel, J., Voss, H. H., Miedaner, T., Korzun, V., Häberle, J., Schweizer, G., Mohler, V., Zimmermann, G., and Hartl, L. 2008. Inheritance of resistance to Fusarium head blight in three European winter wheat populations. Theor. Appl. Genet. 117:1119-1128.

Jiang, G. L., Dong, Y., Shi, J., and Ward, R. W. 2007a. QTL analysis of resistance to Fusarium head blight in the novel wheat germplasm CJ 9306. II. Resistance to deoxynivalenol accumulation and grain yield loss. Theor. Appl. Genet. 115:1043-1052.

Jiang, G. L., Shi, J., and Ward, R. W. 2007b. QTL analysis of resistance to Fusarium head blight in the novel wheat germplasm CJ 9306. I. Resistance to fungal spread. Theor. Appl. Genet. 116:3-13.

Joehanes, R., and Nelson, J. C. 2008. QGene 4.0, an extensible Java QTLanalysis platform. Bioinformatics 24:2788-2789.

Korosteleva, S. N., Smith, T. K., and Boermans, H. J. 2007. Effects of feedborne Fusarium mycotoxins on the performance, metabolism, and immunity of dairy cows. J. Dairy Sci. 90:3867-3873.

Levene, H. 1960. Robust tests for equality of variances. Pages 278-292 in: Contributions to Probability and Statistics: Essays in Honor of Harold Hotelling. I. Olkin, ed. Stanford University Press, Palo Alto, CA.

Liu, S., Hall, M. D., Griffey, C. A., and McKendry, A. L. 2009. Meta-analysis of QTL associated with Fusarium head blight resistance in wheat. Crop Sci. 49:1955-1968.

Liu, S., Pumphrey, M., Gill, B., Trick, H., Zhang, J., Dolezel, J., Chalhoub, B., and Anderson, J. 2008. Toward positional cloning of Fhbl, a major QTL for Fusarium head blight resistance in wheat. Cereal Res. Commun. 36:195-201.

Lorieux, M. 2012. MapDisto: Fast and efficient computation of genetic linkage maps. Mol. Breed. 30:1231-1235.

McCartney, C. A., Somers, D. J., Fedak, G., DePauw, R. M., Thomas, J., Fox, S. L., Humphreys, D. G., Lukow, O., Savard, M. E., McCallum, B. D., and Gilbert, J. 2007. The evaluation of FHB resistance QTLs introgressed into elite Canadian spring wheat germplasm. Mol. Breed. 20:209-221.

McMullen, M., Bergstrom, G., De Wolf, E., Dill-Macky, R., Hershman, D., Shaner, G., and Van Sanford, D. 2012. A unified effort to fight an enemy of wheat and barley: Fusarium head blight. Plant Dis. 96:1712-1728.

McMullen, M., Jones, R., and Gallenberg, D. 1997. Scab of wheat and barley: A re-emerging disease of devastating impact. Plant Dis. 81:1340-1348.

Paillard, S., Schnurbusch, T., Tiwari, R., Messmer, M., Winzeler, M., Keller, B., and Schachermayr, G. 2004. QTL analysis of resistance to Fusarium head blight in Swiss winter wheat (Triticum aestivum L.). Theor. Appl. Genet. 109:323-332.

Parry, D. W., Jenkinson, P., and McLeod, L. 1995. Fusarium ear blight scab in small grain cereals-a review. Plant Pathol. 44:207-238.

Pestka, J. J. 2010. Deoxynivalenol: Mechanisms of action, human exposure, and toxicological relevance. Arch. Toxicol. 84:663-679.

Puri, K. D., and Zhong, S. 2010. The 3ADON population of Fusarium graminearum found in North Dakota is more aggressive and produces a higher level of DON than the prevalent $15 \mathrm{ADON}$ population in spring wheat. Phytopathology 100:1007-1014.

Rawat, N., Pumphrey, M. O., Liu, S., Zhang, X., Tiwari, V. K., Ando, K., Trick, H. N., Bockus, W. W., Akhunov, E., Anderson, J. A., and Gill, B. S. 2016. Wheat $F h b 1$ encodes a chimeric lectin with agglutinin domains and a pore-forming toxin-like domain conferring resistance to Fusarium head blight. Nat. Genet. 48:1576-1580.

Röder, M. S., Korzun, V., Wendehake, K., Plaschke, J., Tixier, M. H., Leroy, P., and Ganal, M. W. 1998. A microsatellite map of wheat. Genetics 149: 2007-2023.

Ruckenbauer, P., Buerstmayr, H., and Lemmens, M. 2001. Present strategies in resistance breeding against scab (Fusarium spp.). Pages 85-95 in: Wheat in a Global Environment. Z. Bedö and L. Láng, eds. Springer, Dordrecht, The Netherlands.

SAS Institute. 2011. SAS/IML 9.3 User's Guide. SAS Institute, Cary NC.

Schmolke, M., Zimmermann, G., Buerstmayr, H., Schweizer, G., Miedaner, T., Korzun, V., Ebmeyer, E., and Hartl, L. 2005. Molecular mapping of 
Fusarium head blight resistance in the winter wheat population Dream/ Lynx. Theor. Appl. Genet. 111:747-756.

Schroeder, H. W., and Christensen, J. J. 1963. Factors affecting resistance of wheat to scab caused by Gibberella zeae. Phytopathology 53:831-838.

Schweiger, W., Steiner, B., Vautrin, S., Nussbaumer, T., Siegwart, G., Zamini, M., Jungreithmeier, F., Gratl, V., Lemmens, M., Mayer, K. F. X., and Berges, H. 2016. Suppressed recombination and unique candidate genes in the divergent haplotype encoding Fhb1, a major Fusarium head blight resistance locus in wheat. Theor. Appl. Genet. 129:1607-1623.

Stack, R. W., Elias, E. M., Fetch, J. M., Miller, J. D., and Joppa, L. R. 2002. Fusarium head blight reaction of Langdon durum-chromosome substitution lines. Crop Sci. 42:637-642.

Stack, R. W., and McMullen, M. P. 1995. A visual scale to estimate severity of Fusarium head blight in wheat. N.D. State Univ. Ext. Publ. PP-1095.

Steiner, B., Lemmens, M., Griesser, M., Scholz, U., Schondelmaier, J., and Buerstmayr, H. 2004. Molecular mapping of resistance to Fusarium head blight in the spring wheat cultivar Frontana. Theor. Appl. Genet. 109:215-224.

Tai, T., and Tanksley, S. D. 1990. A rapid and inexpensive method of isolation of total DNA from dehydrated plant tissue. Plant Mol. Biol. Rep. 8: 297-303.

Waldron, B. L., Moreno-Sevilla, B., Anderson, J. A., Stack, R. W., and Frohberg, R. C. 1999. RFLP mapping of QTL for Fusarium head blight resistance in wheat. Crop Sci. 39:805-811.
Wang, S., Wong, D., Forrest, K., Allen, A., Chao, S., Huang, B. E., Maccaferri, M., Salvi, S., Milner, S. G., Cattivelli, L., Mastrangelo, A. M., Whan, A., Stephen, S., Barker, G., Wieseke, R., Plieske, J., International Wheat Genome Sequencing Consortium, Lillemo, M., Mather, D., Appels, R., Dolferus, R., Brown-Guedira, G., Korol, A., Akhunova, A. R., Feuillet, C., Salse, J., Morgante, M., Pozniak, C., Luo, M.-C., Dvorak, J., Morell, M., Dubcovsky, J., Ganal, M., Tuberosa, R., Lawley, C., Mikoulitch, I., Cavanagh, C., Edwards, K. J., Hayden, M., and Akhunov, E. 2014. Characterization of polyploid wheat genomic diversity using a high-density 90000 single nucleotide polymorphism array. Plant Biotechnol. J. 12:787-796.

Yang, Z., Gilbert, J., Fedak, G., and Somers, D. J. 2005. Genetic characterization of QTL associated with resistance to Fusarium head blight in a doubled-haploid spring wheat population. Genome 48:187-196.

Yu, J. B., Bai, G. H., Zhou, W. C., Dong, Y. H., and Kolb, F. L. 2008. Quantitative trait loci for Fusarium head blight resistance in a recombinant inbred population of Wangshuibai/Wheaton. Phytopathology 98:87-94.

Zhou, W., Kolb, F. L., Bai, G., Shaner, G., and Domier, L. L. 2002. Genetic analysis of scab resistance QTL in wheat with microsatellite and AFLP markers. Genome 45:719-727.

Zhou, W., Kolb, F. L., Yu, J., Bai, G., Boze, L. K., and Domier, L. L. 2004. Molecular characterization of Fusarium head blight resistance in Wangshuibai with simple sequence repeat and amplified fragment length polymorphism markers. Genome 47:1137-1143. 